Y. Matsushima

Nagoya Math. J.

Vol. 46 (1972), 161-173

\title{
REMARKS ON KÄHLER-EINSTEIN MANIFOLDS
}

\author{
YOZO MATSUSHIMA
}

The main purpose of this note is to characterize a compact KählerEinstein manifold in terms of curvature form. The curvature form $\Omega$ is an EndT valued differential form of type $(1,1)$ which represents the curvature class of the manifold. We shall prove that the curvature form of a Kähler metric is the harmonic representative of the curvature class if and only if the Kähler metric is an Einstein metric in the generalized sense (g.s.), that is, if the Ricci form of the metric is parallel. It is well known that a Kähler metric is an Einstein metric in the g.s. if and only if it is locally product (globally, if the manifold is simply connected and complete) of Kähler-Einstein metrics. We obtain an integral formula, involving the integral of the trace of some operators defined by the curvature tensor, which measures the deviation of a Kähler-Einstein metric from a Hermitian symmetric metric. In the final section we shall prove the uniqueness up to equivalence of Kähler-Einstein metrics in a simply connected compact complex homogeneous space. This result was proved by Berger [3] in the case of a complex projective space and our proof is completely different from Berger's.

1. Throughout this paper we shall denote by $M$ a compact Kähler manifold and by $T$ and $T^{*}$ the holomorphic tangent bundle and the holomorphic cotangent bundle of $M$ respectively. The real differentiable tangent bundle of $M$ will be denoted by $T_{R}$. The vector space of smooth sections of a vector bundle $F$ will be denoted by $\Gamma(F)$. A section $X$ of $T$ is a complex vector field of holomorphic type or of type $(1,0)$ and we denote by $\bar{X}$ the conjugate of $X ; \bar{X}$ is a section of the conjugate bundle $\bar{T}$ of $T$. We denote by $\langle$,$\rangle the Hermitian metric in T$, that is, if $X, Y \in \Gamma(T)$, then

$$
\langle X, Y\rangle=g(X, \bar{Y}),
$$

where $g$ denotes the Kähler metric in $M$. We have then

Received October 20, 1971 


$$
X\langle Y, Z\rangle=\left\langle D_{X} Y, Z\right\rangle+\left\langle Y, D_{\bar{X}} Z\right\rangle
$$

for $X, Y, Z \in \Gamma(T)$, where $D$ denotes the operator of the covariant differentiation in the Kähler manifold $M$.

If $\left\{E_{a}\right\}\left(a=1,2, \cdots, n, n=\operatorname{dim}_{c} M\right)$ is a local holomorphic frame field of $T$, then we have [6]

$$
D_{a} E_{b}=\sum_{c} \Gamma_{a b}^{c} E_{c}, D_{a} \bar{E}_{b}=D_{\bar{a}} E_{b}=0
$$

for $a, b=1,2, \cdots, n$, where $D_{a}=D_{E_{a}}$ and $D_{\bar{a}}=D_{\overline{\bar{a}_{a}}}$.

Throughout the paper we shall denote by $E$ the holomorphic vector bundle $E n d T=T^{*} \otimes T$. The covariant derivative $D_{X} A$ of a section $A$ of $E$ is defined to be a section of $E$ such that

$$
\left(D_{X} A\right)(Y)=D_{X}(A(Y))-A\left(D_{X} Y\right), X, Y \in \Gamma\left(T_{R}\right)^{*} .
$$

Let $L$ be an $E$-valued differential $r$-form. The covariant differential $D L$ of $L$ is an $E$-valued differential $(r+1)$-form such that

$$
\begin{aligned}
& (D L)\left(X_{1}, \cdots, X_{r+1}\right)=\sum_{i=1}^{r+1}(-1)^{i+1} D_{X_{i}}\left(L\left(X_{1}, \cdots, \hat{X}_{i}, \cdots, X_{r+1}\right)\right) \\
+ & \sum_{i<j}(-1)^{i+j} L\left(\left[X_{i}, X_{j}\right], X_{1}, \cdots, \hat{X}_{i}, \cdots, \hat{X}_{j}, \cdots, X_{r+1}\right),
\end{aligned}
$$

wereh $X_{1}, \cdots, X_{r+1} \in \Gamma\left(T_{R}\right)$.

If $L$ is of type $(p, q)$, then $D L$ is a sum of a form $D^{\prime} L$ of type $(p+1$, $q$ ) and a form $D^{\prime \prime} L$ of type $(p, q+1)$ :

$$
D L=D^{\prime} L+D^{\prime \prime} L
$$

Let $\left\{E_{a}\right\}$ be a local holomorphic frame field of $T$ defined in an open set $U$ of $M$ and if we write $L$ in the form

$$
L \cdot E_{a}=\sum_{b} L_{a}^{b} \cdot E_{b}
$$

where $L_{a}^{b}$ are differential forms of type $(p, q)$ defined in $U$, then it follows from (1.1) and (1.2) that

$$
D^{\prime \prime} L \cdot E_{a}=\sum_{b} d^{\prime \prime} L_{a}^{b} \cdot E_{b}
$$

In other words, we have

* If $Y \in \Gamma\left(T_{R}\right)$, then we can write $Y$ uniquely in the form $Y=Y_{+}+\bar{Y}_{+}$with $Y_{+} \in \Gamma(T)$ and $A(Y)$ will denote the value of $A$ for the section $Y_{+}$of $T$. 


$$
D^{\prime \prime} L=d^{\prime \prime} L
$$

where $d^{\prime \prime} L$ denote the $E$-valued form defined by $\left(d^{\prime \prime} L_{a}^{b}\right)$. In particular, for a section $A$ of $E$, we have

$$
\left(d^{\prime \prime} A\right)(\bar{X})=D_{\bar{X}} A, \quad X \in \Gamma(T) .
$$

The covariant derivative $D_{X} L$ of $L$ is an $E$-valued $r$-form such that

$$
\left(D_{X} L\right)\left(X_{1}, \cdots, X_{r}\right)=D_{X}\left(L\left(X_{1}, \cdots, X_{R}\right)\right)-\sum_{i} L\left(X_{1}, \cdots, D_{X} X_{i}, \cdots, X_{r}\right)
$$

The operator $D_{X}$ is type preserving and for $r=2$, we have:

$$
(D L)(X, Y, Z)=\left(D_{X} L\right)(Y, Z)+\left(D_{Y} L\right)(Z, X)+\left(D_{Z} L\right)(X, Y) .
$$

2. Let $A$ be a section of $E=E n d T$. The adjoint $A^{*}$ of $A$ is a section of $E$ such that

$$
\langle A X, Y\rangle=\left\langle X, A^{*} Y\right\rangle
$$

for any $X, Y \in \Gamma(T)$. A section $A$ is said to be symmetric if $A=A^{*}$.

Lemma 1. Let $A$ be a symmetric section of $E$. Then $A$ is a holomorphic section if and only if $D A=0$.

Proof. A section $A$ is holomorphic if and only if $d^{\prime \prime} A=D^{\prime \prime} A=0$. Since $D A=D^{\prime} A+D^{\prime \prime} A$ and $D^{\prime} A$ and $D^{\prime \prime} A$ are of type $(1,0)$ and of type $(0,1)$ respectively, if $D A=0$, we get $D^{\prime} A=D^{\prime \prime} A=0$, and so $A$ is holomorphic. Conversely let $A$ be a holomorphic section. Let $\left\{E_{a}\right\}$ be a local orthonormal frame bundle and put $D_{a}=D_{E_{a}}$ and $D_{\bar{a}}=D_{\overline{E_{a}}}, A\left(E_{a}\right)=\sum_{b} A_{a}^{b} E_{b}$. Since $D^{\prime \prime} A=0$, we have $D_{\bar{a}} A_{c}^{b}=0$ for all $a, b, c$. Moreover since $A$ is symmetric, we have $\overline{A_{c}^{b}}=A_{b}^{c}$ and so the conjugate complex of $D_{\bar{a}} A_{c}^{b}$ is $D_{a} A_{b}^{c}$ and hence $D_{a} A_{b}^{c}=0$ for all $a, b, c$. This means that $D^{\prime} A=0$, and so $D A=D^{\prime} A+D^{\prime \prime} A$ $=0$.

Let $L$ be an $E$-valued differential form of type $(1,1)$. We define an $E$-valued differential form $L^{*}$ of type $(1,1)$ by putting

$$
L *(X, \bar{Y})=-L(Y, \bar{X})^{*} .
$$

Lemma 2. Let $L$ be an E-valued differential form of type $(1,1)$ such that $L^{*}$ $=-L$. Then $L$ is $d^{\prime \prime}$-closed if and only if the covariant differential $D L$ of $L$ is zero. 
Proof. As in the proof of Lemma 1, we have to show that $D^{\prime \prime} L=0$ if and only if $D^{\prime} L=0$. By (1.3), $\left(D^{\prime} L\right)\left(E_{a}, E_{b}, \bar{E}_{c}\right)=(D L)\left(E_{a}, E_{b}, \bar{E}_{c}\right)=D_{a} L\left(E_{b}\right.$, $\left.\bar{E}_{c}\right)-\left(D_{b} L\right)\left(E_{a}, \bar{E}_{c}\right)$ and $\left(D^{\prime \prime} L\right)\left(E_{a}, \bar{E}_{b}, \bar{E}_{c}\right)=-\left(D_{\bar{b}} L\right)\left(E_{a}, \bar{E}_{c}\right)+\left(D_{\bar{c}} L\right)\left(E_{a}, \bar{E}_{b}\right)$, where $\left\{E_{a}\right\}$ is a local orthonormal frame field in $T$. If we write $L$ locally in the form

$$
L\left(E_{b}, \bar{E}_{c}\right) E_{k}=\sum_{l} L_{k b \bar{c}}^{l} E_{l}
$$

with $L_{k b \bar{c}}^{l}=L_{k}^{l}\left(E_{b}, \bar{E}_{c}\right)$, then $D^{\prime} L=0$ is equivalent to the set of equations

$$
D_{a} L_{k b \bar{c}}^{l}=D_{b} L_{k a \bar{c}}^{l}
$$

and also $D^{\prime \prime} L=0$ means that

$$
D_{\bar{a}} L_{k b \bar{c}}^{l}=D_{\bar{c}} L_{k b \bar{a}}^{l}
$$

for all $a, b, c, l$ and $k$. Moreover since $L^{*}=-L$, we have

$$
L_{k b \bar{c}}^{l}=\overline{L_{l c \bar{b}}^{k}} .
$$

Then the conjugate complex of $D_{a} L_{k b \bar{c}}^{l}$ is equal to $D_{\bar{a}} L_{l c \bar{b}}^{k}$. It follows from this that $\left(^{*}\right)$ and $\left(^{* *}\right)$ are equivalent and this proves that $D^{\prime} L=0$ if and only if $D^{\prime \prime} L=0$.

Definition. An $E$-valued 2-forms $L$ is called a generalized curvature form if $L$ satisfies the following conditions:

1) $L$ is of type $(1,1)$ and $L^{*}=-L$.

2) $d^{\prime \prime} L=0$.

3) $L(X, \bar{Y}) Z=L(Z, \bar{Y}) X$ for $X, Y, Z \in \Gamma(T)$.

Remark. The third condition on $L$ is called the first Bianchi identity for $L$. By Lemma 2, we can replace the second condition by $D L=0$; that is, $\left(D_{X} L\right)(Y, Z)+\left(D_{Y} L\right)(Z, X)+\left(D_{Z} L\right)(X, Y)=0$ and this last condition is known as the second Bianchi identity. We have included the notion of generalized curvature forms to make clear the relation of our results to those of Nomizu [9].

For each generalized curvature form $L$ we define the Ricci form $K=K_{L}$ of $L$ as follows: $K$ is a differential form of type $(1,1)$ in $M$ defined by

$$
K(X, \bar{Y})=\operatorname{Tr}(L(X, \bar{Y})), X, Y \in \Gamma(T) .
$$

Clearly $K$ is $d^{\prime \prime}$-closed.

There exists a section $S=S_{L}$ of $E$ such that 


$$
\langle S X, Y\rangle=K(X, \bar{Y})
$$

for all $X, Y \in \Gamma(T)$. We call $S$ the Ricci tensor of $L$. Since $L$ satisfies $L=$ $-L^{*}, \cdot S$ satisfies $S=S^{*}$, that is, $S$ is symmetric.

If $\left\{E_{a}\right\}$ is a local orthonormal frame of $T$, then

$$
S=\sum_{a} L\left(E_{a}, \bar{E}_{a}\right)
$$

In fact, $\left\langle S E_{b}, E_{c}\right\rangle=\operatorname{Tr} L\left(E_{b}, \bar{E}_{c}\right)=\sum_{a}\left\langle L\left(E_{b}, \bar{E}_{c}\right) E_{a}, E_{a}\right\rangle$ and by the condition 3) on $L, L\left(E_{b}, \bar{E}_{c}\right) E_{a}=L\left(E_{a}, \bar{E}_{c}\right) E_{b}$. By the condition $L=-L^{*}$ and by $3)$, we have $\left\langle L\left(E_{a}, \bar{E}_{\mathrm{c}}\right) E_{b}, E_{a}\right\rangle=\left\langle E_{b}, L\left(E_{\mathrm{c}}, \bar{E}_{a}\right) E_{a}\right\rangle=\left\langle E_{b}, L\left(E_{a}, \bar{E}_{a}\right) E_{\mathrm{c}}\right\rangle=\left\langle L\left(E_{a}\right.\right.$, $\left.\left.\bar{E}_{a}\right) E_{b}, E_{c}\right\rangle$ and hence $\left\langle S E_{b}, E_{c}\right\rangle=\sum_{a}\left\langle L\left(E_{a}, \bar{E}_{a}\right) E_{b}, E_{c}\right\rangle$ which proves (2.2).

3. For any two $E$-valued differential forms $L$ and $L^{\prime}$ of type $(p, q)$, we denote by $\left\langle L, L^{\prime}\right\rangle$ their scalar product defined by the Hermitian metric in $T$ and by $\left(L, L^{\prime}\right)$ their inner product i.e.,

$$
\left(L, L^{\prime}\right)=\int_{M}\left\langle L, L^{\prime}\right\rangle d v,
$$

where $d v$ denotes the volume element of the Kähler manifold $M$. The adjoint of the operator $d^{\prime \prime}$ with respect to the inner product will be denoted by $\delta^{\prime \prime}$. If $L$ is of type $(p, q+1)$, then $\delta^{\prime \prime} L$ is of type $(p, q)$ and $\left(\delta^{\prime \prime} L, L^{\prime}\right)$ $=\left(L, d^{\prime \prime} L^{\prime}\right)$ holds for any $L^{\prime}$ of type $(p, q)$.

The complex Laplacian $\square^{\prime \prime}$ is the operator defined by

$$
\square^{\prime \prime}=d^{\prime \prime} \delta^{\prime \prime}+\delta^{\prime \prime} d^{\prime \prime} \text {. }
$$

The operator $\square^{\prime \prime}$ is type preserving and an E-valued form $L$ is said to be harmonic if $\square^{\prime \prime} L=0$.

Let $D^{p, q}(E)$ denote the complex vector space of all $E$-valued forms of type $(p, q)$ and let

$$
D^{p}(E)=\sum_{q} D^{p, q}(E)
$$

Then $D^{p}(E)$ is a complex with coboundary operator $d^{\prime \prime}$; the cohomology group of the complex $D^{p}(E)$ is denoted by $H^{p, q}(M, E)$. By the DolbeaultSerre theorem, we have a canonical isomorphism

$$
H^{p, q}(M, E) \cong H^{q}\left(M, \underline{\left.\left(\stackrel{p}{\wedge} T^{*}\right) \otimes E\right)}\right.
$$

where, for any holomorphic vector bundle $F, \underline{F}$ denotes the shief of germs 
of holomorphic sections.

Let $\Omega$ be the curvature form of the Kähler metric in $M$. Then $\Omega$ is an $E$-valued form of type (1.1) and $d^{\prime \prime} \Omega=0$. Hence $\Omega$ represents a cohomology class in $H^{1,1}(M, E)=H^{1}\left(M, T^{*} \otimes E\right)$, where $E=T^{*} \otimes T$. The cohomology class is independent of the choice of Kähler metric in $M$. We shall call this cohomology class the curvature class of $M$. As a matter of fact, the curvature class may be defined for any compact complex manifold; it is defined to be the cohomology class in $H^{1,1}(M, E)$ represented by the curvature form of a connection of type $(1,1)$ in $T$, for more details see Atiyah [1]. Moreover, the curvature class of $M$ is zero if and only if $T$ has a holomorphic linear connection [1].

Let $L$ be an $E$-valued form of type $(1,1)$. Then $\delta^{\prime \prime} L$ is an $E$-valued form of type $(1,0)$. By an easy computation and by the Stokes theorem, we obtain the following formula. Let $\left\{E_{a}\right\}$ be a local orthonormal frame field of $T$. Then

$$
\left(\delta^{\prime \prime} L\right)\left(E_{a}\right)=\sum_{b}\left(D_{b} L\right)\left(E_{a}, \bar{E}_{b}\right)
$$

for all $a=1,2, \cdots, n$.

Lemma 3. Let $L$ be a generalized curvature tensor and $S$ the Ricci tensor of L. Then we have

$$
\delta^{\prime \prime} L=D^{\prime} S
$$

Proof. Let $\left\{E_{a}\right\}$ be a local orthonormal frame field of $T$. By Lemma 2 we have $D L=0$ and hence $\left(D_{b} L\right)\left(E_{a}, \bar{E}_{b}\right)=\left(D_{a} L\right)\left(E_{b}, \bar{E}_{b}\right)$ and by (3.1) we obtain $\left(\delta^{\prime \prime} L\right)\left(E_{a}\right)=\sum_{b}\left(D_{a} L\right)\left(E_{b}, \bar{E}_{b}\right)=\sum_{b} D_{a}\left(L\left(E_{b}, \bar{E}_{b}\right)\right)-\sum_{b} L\left(D_{a} E_{b}, \bar{E}_{b}\right)-\sum_{b} L\left(E_{b}\right.$, $\left.D_{a} \bar{E}_{b}\right)$. On the other hand, by (2.2), $\left(D^{\prime} S\right)\left(E_{a}\right)=D_{a} S=\sum_{b} D_{a}\left(L\left(E_{b}, E_{\bar{b}}\right)\right)$ and hence

$$
\left(\delta^{\prime \prime} L\right)\left(E_{a}\right)=\left(D^{\prime} S\right)\left(E_{a}\right)-\sum_{b}\left\{L\left(D_{a} E_{b}, \bar{E}_{b}\right)+L\left(E_{b}, D_{a} \bar{E}_{b}\right)\right\} .
$$

We show that the second term of the right hand side is zero. To see this let $D_{a} E_{b}=\sum_{c} \Gamma_{c, b}^{c} E_{c}$ and $D_{\bar{a}} E_{b}=\sum_{c} \Gamma_{a}^{c} E_{c}$. Then $D_{a} \bar{E}_{b}=\sum_{c} \overline{\Gamma_{c}^{c}, b} \bar{E}_{c}$. It follows from

$$
0=D_{a}\left\langle E_{b}, E_{d}\right\rangle=\left\langle D_{a} E_{b}, E_{d}\right\rangle+\left\langle E_{b}, D_{\bar{a}} E_{d}\right\rangle \text { that } \Gamma_{a b}^{c}+\bar{\Gamma}_{\bar{a} c}^{b}=0
$$

for all $a, b, c$ and hence 


$$
\begin{gathered}
\sum_{b}\left\{L\left(D_{a} E_{b}, \bar{E}_{b}\right)+L\left(E_{b}, D_{a} \bar{E}_{b}\right)\right\}=\sum_{b, c} \Gamma_{a b}^{c} L\left(E_{c}, \bar{E}_{b}\right) \\
+\sum_{b, c} \bar{\Gamma}_{a, b}^{c} L\left(E_{b}, \bar{E}_{c}\right)=\sum_{b, c}\left(\Gamma_{a b}^{c}+\bar{\Gamma}_{a c}^{b}\right) L\left(E_{c}, \bar{E}_{b}\right)=0 .
\end{gathered}
$$

This proves that $\left(\delta^{\prime \prime} L\right)\left(E_{a}\right)=\left(D^{\prime} S\right)\left(E_{a}\right)$ for all $a$ and hence $\delta^{\prime \prime} L=D^{\prime} S$.

Proposition. Let $E$ be a generalized curvature form of a compact Kähler manifold $M$. Then $L$ is a harmonic E-valued form, where $E=E n d T$, if and only if the Ricci tensor $S_{L}$ of $L$ is a holomorphic section of $E$. Moreover $S_{L}$ is a holomorphic section of $E$ if and only if $S_{L}$ is parallel.

Proof. Since $d^{\prime \prime} L=0, L$ is harmonic if $\delta^{\prime \prime} L=0$. By Lemma 3, we have $\delta^{\prime \prime} L=D^{\prime} S$ and $D S=D^{\prime} S+D^{\prime \prime} S, d^{\prime \prime} S=D^{\prime \prime} S$. Since $S$ is symmetric, we have $d^{\prime \prime} S=0$ if and only if $D S=0$ by Lemma 1 and this proves our assertion in the proposition.

A Kähler metric is said to be a Kähler-Einstein metric in the generalized sense (g.s.) if the Ricci form is parallel. Since the curvature form is obviously a generalized curvature form, we obtain as a special case of the proposition the following:

Theorem 1. A compact Kähler manifold is a Kähler-Einstein manifold in the g.s. if and only if the curvature form is a harmonic E-valued form, where $E=E n d T$.

Remark. According to Atiyah [1] the curvature class of a complex manifold is zero if and only if the holomorphic tangent bundle $T$ has a holomorphic linear connection. Therefore if a compact Kähler-Einstein manifold in the g.s. $M$ admits a holomorphic linear connection, then the curvature form $\Omega$ of $M$ is zero by Theorem 1 and so $M$ is locally flat. It is also known that if the second Chern class of a compact Kähler-Einstein manifold $M$ is zero, then $M$ is locally flat (Apt [2]). A complex manifold $M$ is said to be a complex affine manifold if $M$ admits a holomorphic linear connection whose curvature and torsion are zero. There is no known example of compact complex affine Kähler manifold which is not flat ( $c f[11]$ ).

4. Let $L$ be any $E$-valued form of type $(1,1)$. The components of $L$ with respect to a local frame field of $T$ will be denoted by $L_{k a \bar{b}}^{l}(a, b, k, l$ $=1,2, \cdots, n)$; namely if $\left\{E_{k}\right\}$ is a local frame field and $L\left(E_{a} \bar{E}_{b}\right) E_{k}=$ $\sum_{l} L_{k}^{l}\left(E_{a}, \bar{E}_{b}\right) E_{l}$, then $L_{k a \bar{b}}^{l}=L_{k}^{l}\left(E_{a}, \bar{E}_{b}\right)$. If $\left\{E_{k}\right\}$ is orthonormal we write $L_{\bar{l} k a \bar{b}}$ in place of $L_{k a \bar{b}}^{l}$. Let $\Omega$ be the curvature form of $M$. We denote the 
components of $\Omega$ by $R_{k a \bar{b}}^{l}$ so that we have

$$
\Omega \cdot E_{k}=\sum_{l}\left(\sum_{a, b} R_{k a, \bar{b}}^{l} \omega^{a} \wedge \bar{\omega}^{b}\right) E_{l},
$$

where $\left\{\omega^{a}\right\}$ denotes the local coframe bundle such that $\omega^{a}\left(E_{b}\right)=\delta_{b}^{a}$. The components of the Ricci tensor of $\Omega$ will be denoted by $K_{k}^{l} ; K_{\bar{k}}^{\bar{l}}$ denotes the conjugate complex of $K_{k}^{l}$.

Then the components of the $E$-valued form $\square^{\prime \prime} L$ are given by the following formula:

$$
\begin{aligned}
\left(\square^{\prime \prime} L\right)_{k a \bar{b}}^{l}= & -\sum_{c} D^{\bar{c}} D_{\bar{c}} L_{k a \bar{b}}^{l}+\sum_{c} K_{\bar{b}}^{\bar{c}} L_{k a \bar{c}}^{l} \\
& -\sum_{c, \overline{d, i}} g^{\bar{c} d} L_{k i \bar{c}}^{l} R_{d a \bar{b}}^{i} \\
& +\sum_{c, \bar{d}, i} g^{\bar{c} d}\left\{R_{i d \bar{b}}^{l} L_{k a \bar{c}}^{i}-L_{a i \bar{c}}^{l} R_{k d \bar{b}}^{i}\right\}
\end{aligned}
$$

where $D^{\bar{c}}=g^{\bar{c} d} D_{d}$. In particular, for $L=\Omega$, we obtain from the first Bianchi identity $R_{k i \bar{c}}^{l}=R_{i k \bar{c}}^{l}$,

$$
\begin{aligned}
\left(\square^{\prime \prime} \Omega\right)_{k a, \bar{b}}^{l}= & -\sum_{c} D^{\bar{c}} D_{\bar{c}} R_{k a \bar{b}}^{l}+\sum_{c} K_{\bar{b}}^{\bar{c}} R_{k a \bar{c}}^{l} \\
& +\sum_{c, d, i} g^{\bar{c} d} R_{i a \bar{b}}^{l} R_{k a \bar{c}}^{i} \\
& -\sum_{c, \overline{d, i}} g^{\bar{c} d}\left\{R_{i k \bar{c}}^{l} R_{d a \bar{b}}^{i}+R_{i a \bar{c}}^{l} R_{d k \bar{b}}^{i}\right\} .
\end{aligned}
$$

Suppose now that the metric is Kähler-Einstein. Then we have

$$
K_{c \bar{b}}=\rho \cdot g_{c \bar{b}}, \quad(b, c=1, \cdots, n)
$$

where $\rho$ is a constant. Assume that our local frame is orthonormal. Then since $\square^{\prime \prime} \Omega=0$ by Theorem 1 , we obtain

$$
\begin{aligned}
0= & -\sum_{c} D_{c} D_{\bar{c}} R_{k a \bar{b}}^{l}+\rho R_{k a \bar{b}}^{l} \\
& +\sum_{c, i} R_{\bar{l} i c \bar{b}} R_{\bar{i} k a \bar{c}} \\
& -\sum_{c, i}\left\{R_{\bar{l} i k \bar{c}} R_{\bar{l} c a \bar{b}}+R_{\left.\bar{l} i a \bar{c} \bar{c} R_{\bar{i} c k \bar{b}}\right\}}(l, k, a, b=1,2, \cdots, n)\right.
\end{aligned}
$$

Hence we get

$$
\left\langle\square^{\prime \prime} \Omega, \Omega\right\rangle=\delta \theta+\langle D R, D R\rangle+\rho|\Omega|^{2}+F-2 G=0,
$$

where $\theta$ is a differential form of type $(0,1)$ defined by $\theta\left(\bar{E}_{c}\right)=\left\langle D_{\bar{c}} \Omega, \Omega\right\rangle, D R$ denotes the covariant differential of the curvature tensor (note that this is 
distinct from the covariant differential of $\Omega$ which is zero), $|\Omega|$ is the length of the curvature form $\Omega$ and $F$ and $G$ are functions on $M$ defined as follows:

$$
\begin{aligned}
& F=\sum R_{\bar{l} i c \bar{b}} R_{\bar{i} k a \bar{c}} R_{\bar{k} l b \bar{a}}, \\
& G=\sum R_{\bar{l} i k \bar{c} \overline{\bar{c}} c a \bar{b}} R_{\bar{k} l b \bar{\alpha}},
\end{aligned}
$$

where sum extends over all indices.

Integrating over $M$, we obtain

$$
(D R, D R)+\rho(\Omega, \Omega)+\int_{M} F d v-2 \int_{M} G d v=0 .
$$

Since $(D R, D R) \geqq 0$ and $(D R, D R)=0$ means that $M$ is Hermitian symmetric, we see that

$$
2 \int_{M} G d v-\int_{M} F d v-\rho(\Omega, \Omega) \geqq 0
$$

and the equality holds if and only if $M$ is Hermitian symmetric.

The functions $F$ and $G$ can be interpreted as follows by introducing two kinds of operator defined by curvature tensor. These two curvature operators appeared in the paper [5]. Let $\left\{E_{k}\right\}$ be any local orthonormal frame of $T$. The linear operator

$$
H: T \otimes T \rightarrow T \otimes T
$$

is defined by

$$
H: E_{a} \otimes E_{b} \rightarrow \sum_{k, l} R_{\bar{k} a b \bar{l}} E_{k} \otimes E_{l}
$$

Let

$$
H_{(k \imath)(a b)}=R_{\bar{k} a b \bar{l}} \cdot
$$

Then

$$
H\left(E_{a} \otimes E_{b}\right)=\sum_{k, l} H_{(k l)(a b)}\left(E_{k} \otimes E_{l}\right) .
$$

By the first Bianchi identity $R_{\bar{k} a b \bar{l}}=R_{\bar{k} b a \bar{l}}=R_{\bar{l} a b \bar{k}}$ we have

$$
H_{(k l)(a b)}=H_{(k l)(b a)}=H_{(l k)(a b)} .
$$

It follows then that $H\left(E_{a} \otimes E_{b}-E_{b} \otimes E_{a}\right)=0$ and that $H\left(E_{a} \otimes E_{b}+E_{b} \otimes E_{a}\right)$ is a symmetric tensor. Moreover since we have $R_{\bar{k} a b \bar{l}}=\bar{R}_{\bar{a} k l \bar{b}}$, the matrix

$$
H=\left(H_{(k l)(b a)}\right)
$$

is Hermitian. Now we obtain: 


$$
\begin{aligned}
|\Omega|^{2} & =\sum R_{\bar{k} a b \bar{l}} \bar{R}_{\bar{k} a b \bar{l}}=\sum H_{(k l)(a b)} \bar{H}_{(k l)(a b)}=\operatorname{Tr} H^{2}, \\
F & =\sum R_{\bar{k} a b \bar{l}} R_{\bar{a} c a \bar{b}} R_{\bar{c} k l \bar{l}} \\
& =\sum H_{(k l)(a b)} H_{(a b)(c d)} H_{(c d)(k l)}=\operatorname{Tr} H^{3} .
\end{aligned}
$$

The operator

$$
P: T \otimes T \rightarrow T \otimes T
$$

is defined by

$$
P: E_{a} \otimes E_{b} \rightarrow \sum_{k l} R_{\bar{k} l a \bar{\iota}} E_{k} \otimes E_{l}
$$

Let

$$
P_{(k l)(a b)}=R_{\bar{k} l a \bar{b}}
$$

Then

$$
P\left(E_{a} \otimes E_{b}\right)=\sum_{k l} P_{(k l)(a b)} E_{k} \otimes E_{l}
$$

Since $R_{\bar{k} l a \bar{b}}=R_{a \bar{b} \bar{k} l}=\bar{R}_{\bar{a} b k \bar{l}}$, we have $P_{(k l)(a b)}=\bar{P}_{(a b)(k l)}$ and hence the matrix

$$
P=\left(P_{(k l)(a b)}\right)
$$

is Hermitian. Moreover we have:

$$
\begin{aligned}
P_{(k l)(a b)} & =P_{(b a)(l k)}, \quad P_{(k l)(a b)}=P_{(k a)(l b)} \\
G & =\sum R_{\overline{l i k} \bar{c}} R_{\bar{i} c a \bar{b}} R_{\bar{k} l b \bar{a}} \\
& =\sum P_{(l i)(k c)} P_{(i c)(a b)} P_{(k l)(b a)} \\
& =\sum P_{(l k)(i c)} P_{(i c)(a b)} P_{(a b)(l k)}=\operatorname{Tr} P^{3} .
\end{aligned}
$$

Thus we get $G=\operatorname{Tr} P^{3}$

Summing up we obtain the following

Theorem 2. Let $M$ be a compact Kähler-Einstein manifold. Then we have

$$
2 \int_{M} \operatorname{Tr} P^{3} d v-\rho \int_{M} \operatorname{Tr} H^{2} d v-\int_{M} \operatorname{Tr} H^{3} d v \geqq 0
$$

and the equality holds only when $M$ is Hermitian symmetric. In the above inequality $\rho$ is a constant such that $\rho g_{a, \bar{b}}=K_{a, \bar{b}}$ and $|\Omega|^{2}=\operatorname{Tr} H^{2}=\operatorname{Tr} P^{2}$.

Remark. We have $\operatorname{Tr} P=\operatorname{Tr} H=\sum_{a, b} R_{\bar{a} a, b \bar{b}}=\sum_{a} K_{\bar{a} a}=n \rho$ and hence

$$
n \rho=\operatorname{Tr} P=\operatorname{Tr} H, n=\operatorname{dim}_{c} M \text {. }
$$


We consider now the special case where $n=2$. Then we have

$$
R_{\overline{1} 1 a \bar{b}}+R_{\overline{2} 2 a \bar{b}}=R_{\bar{a} b 1 \bar{T}}+R_{\bar{a} b 2 \overline{2}}=\rho \tilde{\delta}_{a b} \quad(a, b=1,2) .
$$

We define a linear operator $J: T \otimes T \rightarrow T \otimes T$ by

$$
J\left(E_{1} \otimes E_{b}\right)=E_{2} \otimes E_{b}, J\left(E_{2} \otimes E_{b}\right)=-E_{1} \otimes E_{b} \quad(b=1,2) .
$$

Then from (4.2) and from the first Bianchi identity we obtain

$$
J^{-1} P J=\rho I-\bar{H},
$$

where $I$ is the identity operator $T \otimes T \rightarrow T \otimes T$ and $\bar{H}$ denotes the operator $T \otimes T \rightarrow T \otimes T$ whose matrix is the conjugate complex of $H$. It follows easily from (4.3) that

$$
\left\{\begin{array}{l}
\operatorname{Tr} P^{3}=-2 \rho^{3}+3 \rho \operatorname{Tr} H^{2}-\operatorname{Tr} H^{3} \\
\operatorname{Tr} H^{3}=-2 \rho^{3}+3 \rho \operatorname{Tr} P^{2}-\operatorname{Tr} P^{3} .
\end{array}\right.
$$

We obtain from (4.1) the following formulae

$$
\begin{aligned}
& 3 \int_{M} \operatorname{Tr} P^{3} d v-4 \rho \int_{M} \operatorname{Tr} P^{2} d v+\frac{\rho}{2} \int_{M}(\operatorname{Tr} P)^{2} d v \geqq 0, \\
& 3 \int \operatorname{Tr} H^{3} d v-5 \rho \int_{M} \operatorname{Tr} H^{2} d v+\rho \int_{M}(\operatorname{Tr} H)^{2} d v \leqq 0 .
\end{aligned}
$$

The Chern numbers of $M$ are given by the formulae

$$
c_{1}^{2}[M]=\frac{1}{8 \pi^{2}} \int_{M}(T r H)^{2} d v, \quad c_{2}[M]=\frac{1}{8 \pi^{2}} \int_{M} T r H^{2} d v,
$$

and we have also $\operatorname{Tr} P=\operatorname{Tr} H, \operatorname{Tr} P^{2}=\operatorname{Tr} H^{2}, 2 \rho=\operatorname{Tr} H$. We can express (4.4) and $\left(4.4^{\prime}\right)$ in the form

$$
\begin{aligned}
& \frac{3}{8 \pi^{2}} \int_{M} \operatorname{Tr} P^{3} d v-4 \rho \cdot c_{2}[M]+\frac{1}{2} \rho c_{1}^{2}[M] \geqq 0, \\
& \frac{3}{8 \pi^{2}} \int_{M} \operatorname{Tr} H^{3} d v-5 \rho c_{2}[M]+\rho c_{1}^{2}[M] \leqq 0,
\end{aligned}
$$

and the equality holds if and only if $M$ is Hermitian symmetric. The geometric meaning of the integral of $\operatorname{Tr} P^{3}$ and $\operatorname{Tr} H^{3}$ is not known.

Let $\mu_{1}, \mu_{2}, \mu_{3}$ and $\mu_{4}$ be the eigen-values of $P$. Since $H\left(E_{a} \otimes E_{b}+E_{b}\right.$ $\left.\otimes E_{a}\right)=0$, one of the eigen-values of $H$ is equal to zero. Then it follows from (4.3) that $\rho$ is an eigen-value of $P$ and we put $\mu_{4}=\rho$. Then we have $\rho=\mu_{1}+\mu_{2}+\mu_{3}$ because $2 \rho=\operatorname{Tr} P$ and we can express the function 
$f=3 \operatorname{Tr} P^{3}-4 \rho \operatorname{Tr} P^{2}+\frac{\rho}{2}(\operatorname{Tr} P)^{2}$ in the form*

$$
f=-\mu_{1}\left(\mu_{2}-\mu_{3}\right)^{2}-\mu_{2}\left(\mu_{3}-\mu_{1}\right)^{2}-\mu_{3}\left(\mu_{1}-\mu_{2}\right)^{2} .
$$

For each unit tangent vector at $x \in M$ let $\sigma(u)$ denote the holomorphic sectional curvature for the complex line spanned by $u$, that is,

$$
\sigma(u)=4 \sum R_{\bar{k} l a \bar{b}} \bar{U}^{k} U^{l} \bar{U}^{b} U^{a}=4\langle H(U \otimes U), U \otimes U\rangle .
$$

We can choose a local orthonormal frame field $\left\{E_{1}, E_{2}\right\}$ in such a way that $\sigma\left(E_{1}(x)\right)=4 R_{\overline{1}_{11 \overline{1}}}=\operatorname{Max}_{|u|=1} \sigma(u)$ and that $R_{\overline{1} 1 a \bar{b}}=0$ for $a \neq b$. ([3]). Then $R_{\overline{1}_{1} a \bar{b}}$ $+R_{\overline{2} 2 a \bar{b}}=\rho \delta_{a b}$ and hence $R_{\overline{2} 2 a \bar{b}}=0$ for $a \neq b$ and $R_{\overline{2} 21 \overline{1}}=R_{\overline{1} 12 \overline{2}}=\rho-R_{\overline{1} 11 \overline{1}}$. Moreover $R_{\overline{2} 2 \overline{2}}=\rho-R_{\overline{\mathrm{T}}_{12 \overline{2}}}=\rho-\left(\rho-R_{\overline{\mathrm{T} 11 \overline{\mathrm{I}}}}\right)=R_{\overline{\mathrm{I} 11 \overline{\mathrm{T}}}}$. Using these relations and the Bianchi identity, we can express the eigen-values of $P$ easily by the components of curvature tensor and we get

$$
\mu_{1}=-R_{\overline{1} 12 \overline{2}}, \mu_{2}=R_{\overline{1} 12 \overline{2}}+\left|R_{\overline{1} 22 \overline{1}}\right|, \mu_{3}=R_{\overline{1} 12 \overline{2}}-\left|R_{\overline{1} 22 \overline{1}}\right| \text {. }
$$

If we assume that the bisectional curvatures [7] are nonnegative, we can show that $f \leqq 0$ and hence $f=0$ by (4.4). Then $M$ is symmetric and isometric either to $P^{2}$ or $P^{1} \times P^{1}$. This result was found by Berger [3].

5. Let $M$ be a compact simply connected complex manifold. We assume that $M$ is homogeneous and that $M$ has a Kähler metric. Let $G_{C}$ be the identity component of the group of all holomorphic transformations of $M$ and let $G_{0}$ be a maximal compact subgroup of $G_{C}$. It is well-known that $G_{0}$ is also transitive on $M$ and $G_{0}$ is semi-simple and $G_{C}$ is the complexification of $G_{C}$ (cf. [4]). Since $G_{0}$ is compact and $M$ has a Kähler metric, we may assume that $M$ has a $G_{0}$-invariant Kähler metric. Let $\nu=i^{n 2} F d z^{1}$ $\wedge \cdots \wedge d z^{n} \wedge d \bar{z}^{1} \wedge \cdots \wedge d \bar{z}^{n}$ be any $G_{0}$-invariant volume element in $M$. Then the differential form $\gamma_{0}=2 d^{\prime} d^{\prime \prime} \log F$ is well defined and $\gamma_{0}$ does not depend on the choice of $\nu$. Moreover $\gamma_{0}$ is $G_{0}$-invariant and it was proved by Koszul [8] that $\gamma_{0}$ is negative definite. Since the Ricci form of any $G_{0}$ invariant in $M$ coincides with $-\gamma_{0}$ by a well-known formula (cf. [6]), every $G_{0}$-invariant Kähler metric has the same Ricci form which is equal to $-\gamma_{0}$. Let $\omega_{0}=-\gamma_{0}$. Then $\omega_{0}$ is positive definite and $G_{0}$-invariant and so the fundamental form of a $G_{0}$-invariant Kähler metric $g_{0}$. Then $g_{0}$ is KählerEinstein. We call $g_{0}$ the canonical Kähler metric in $M$ with respect to $G_{0}$.

Theorem 3. Any Kähler-Einstein metric $g$ on $M$ is equivalent to $g_{0}$; i.e., there exists a holomorphic transformation $\varphi$ of $M$ and a positive constant a such that

* I owe this formula and (4.3) to B. Smyth. 


$$
a g_{0}=\varphi^{*} g .
$$

Proof. We may identify the Lie algebra of $G_{C}$ with the Lie algebra a of all holomorphic vector fields of $M$. Then the subalgebra of a corresponding to $G_{0}$ is identified with the Lie algebra $\mathfrak{g}_{0}$ of all Killing vector field of $\left(M, g_{0}\right)$. Since $G_{C}$ is complex semi-simple and $G_{0}$ is maximal compact in $G_{C}, \mathfrak{a}$ is the complexification of $g_{0}$ and since the complex structure in the Lie algebra $\mathfrak{a}$ is given by the tensor $J$ of the complex structure in $M$, we have $\mathfrak{a}=g_{0}+J g_{0}$. Now let $g$ be any Kähler-Einstein metric in $M$ and $g$ the Lie algebra of Killing vector fields of $(M, g)$. Since $g$ is a compact subalgebra of $\mathfrak{a}$ and since $g_{0}$ is a maximal compact subalgebra of $\mathfrak{a}$, there exists an element $\varphi \in G_{C}$ such that $A d\left(\varphi^{-1}\right) \mathfrak{g} \subset g_{0}$. On the other hand, since $(M, g)$ is Kähler-Einstein, it is known that $\mathfrak{a}=\mathfrak{g}+J \mathfrak{g}$ ([10]). It follows then that $\operatorname{dim} \mathrm{g}=\operatorname{dim} \mathrm{g}_{0}$ and hence $A d\left(\varphi^{-1}\right) \mathrm{g}=\mathrm{g}_{0}$ and $\mathrm{g}$ is maximal compact. The connected subgroup $G$ of $G_{C}$ corresponding to $g$ is a maximal compact subgroup of $G_{C}$ and $\varphi^{-1} G \varphi=G_{0}$. Since $g$ is $G$-invariant, $\varphi^{*} g$ is $G_{0}$-invariant and obviously $\varphi^{*} g$ is also Kähler-Einstein. The fundamental form of $\varphi^{*} g$ is then proportional to $\omega_{0}$ and this proves the theorem.

\section{BiBLIOGRAPHY}

[1] Atiyah, M.F., Complex analytic connections in fibre bundles Trans. Amer. Math. Soc. 85 (1957), 181-207.

[2] Apte, M., Sur certaines classes caractéristiques des variétés kaehleriennes compactes, C.R. Acad. Sci. Paris 240 (1955), 149-151.

[ 3 ] Berger, M., Sur les variétés d'Einstein compactes, C.R. III ${ }^{e}$ Reunion Math. Expression latine, Namur (1965), 35-55.

[4] Borel, A. and Remmert, R., Über kompakte homogene Kählersche Mannigfaltigkeiten, Math. Ann. 145 (1962), 429-439.

[5] Calabi, E. and Vesentini, E., On compact, locally symmetric Kaehler manifolds, Ann. of Math. 71 (1960), 472-507.

[6] Kobayashi, S. and Nomizu, K., Foundations of differential geometry, II, Interscience Publishers, 1969.

[ 7 ] Goldberg, S.I. and Kobayashi, S., On holomorphic bisectional curvature, J. Diff. Geom. 1 (1967), 225-233.

[8] Koszul, J. L., Sur la forme hermitienne cononique des espaces homogènes complexes, Canad. J. Math. 7 (1955), 562-576.

[9] Nomizu, K., On the spaces of generalized curvature tensor fields and second fundamental forms, to appear.

[10] Matsushima, Y., Sur la structure du groupe d'homéomorphismes analytiques d'une certaine variété kaehlerienne, Nagoya Math. J. 11 (1957), 145-150.

[11] Vitter, A. L., Affine Structures on compact complex manifolds, Thesis, Princeton Univ. (1970).

Department of Mathematics, University of Notre Dame. 